\title{
Effect of exercise training and carvedilol treatment on cardiac function and structure in mice with sympathetic hyperactivity- induced heart failure
}

\author{
A. Medeiros ${ }^{1 *}$, A.S. Vanzelli ${ }^{*}$, K.T. Rosa ${ }^{2}$, M.C. Irigoyen ${ }^{2}$ and P.C. Brum ${ }^{1}$ \\ 1Departamento de Biodinâmica do Movimento do Corpo Humano, Escola de Educação Física e Esporte, \\ ${ }^{2}$ Instituto do Coração, Faculdade de Medicina, Universidade de São Paulo, São Paulo, SP, Brasil \\ Correspondence to: P.C. Brum, Departamento de Biodinâmica do Movimento do Corpo Humano, Escola \\ de Educação Física e Esporte, USP, Av. Prof. Mello Moraes, 65, 05508-900 São Paulo, SP, Brasil \\ Fax: +55-11-3813-5921. E-mail: pcbrum@usp.br
}

\begin{abstract}
The present investigation was undertaken to study the effect of $\beta$-blockers and exercise training on cardiac structure and function, respectively, as well as overall functional capacity in a genetic model of sympathetic hyperactivity-induced heart failure in mice $\left(\alpha_{2 \mathrm{~A}} / \alpha_{2 \mathrm{C}} \mathrm{ArKO}\right) . \alpha_{2 \mathrm{~A}} / \alpha_{2 \mathrm{C}} \mathrm{ArKO}$ and their wild-type controls were studied for 2 months, from 3 to 5 months of age. Mice were randomly assigned to control $(\mathrm{N}=45)$, carvedilol-treated $(\mathrm{N}=29)$ or exercise-trained $(\mathrm{N}=33)$ groups. Eight weeks of carvedilol treatment (38 mg/kg per day by gavage) or exercise training (swimming sessions of $60 \mathrm{~min}, 5$ days/week) were performed. Exercise capacity was estimated using a graded treadmill protocol and HR was measured by tail cuff. Fractional shortening was evaluated by echocardiography. Cardiac structure and gastrocnemius capillary density were evaluated by light microscopy. At 3 months of age, no significant difference in fractional shortening or exercise capacity was observed between wild-type and $\alpha_{2 \mathrm{~A}} /$ $\alpha_{2 \mathrm{C}}$ ArKO mice. At 5 months of age, all $\alpha_{2 \mathrm{~A}} / \alpha_{2 \mathrm{C}}$ ArKO mice displayed exercise intolerance and baseline tachycardia associated with reduced fractional shortening and gastrocnemius capillary rarefaction. In addition, $\alpha_{2 \mathrm{~A}} / \alpha_{2 \mathrm{C}} \mathrm{ArKO}$ mice presented cardiac myocyte hypertrophy and ventricular fibrosis. Exercise training and carvedilol similarly improved fractional shortening in $\alpha_{2 \mathrm{~A}} /$ $\alpha_{2 C}$ ArKO mice. The effect of exercise training was mainly associated with improved exercise tolerance and increased gastrocnemius capillary density while $\beta$-blocker therapy reduced cardiac myocyte dimension and ventricular collagen to wildtype control levels. Taken together, these data provide direct evidence for the respective beneficial effects of exercise training and carvedilol in $\alpha_{2 \mathrm{~A}} / \alpha_{2 \mathrm{C}} \mathrm{ArKO}$ mice preventing cardiac dysfunction. The different mechanisms associated with beneficial effects of exercise training and carvedilol suggest future studies associating both therapies.
\end{abstract}

Key words: Heart failure; Exercise training; Carvedilol treatment; Ventricular function; Cardiac remodeling; $\alpha_{2 A} / \alpha_{2 C} A r K O$ mice

*These authors contributed equally to this study.

Research supported by FAPESP (\#02/04588-8). A. Medeiros had a Doctoral scholarship from FAPESP (\#2004/00745-7). A.S. Vanzelli had a Master scholarship from CNPq (\#131016/2003). P.C. Brum holds a scientific productivity fellowship from CNPq.

Received November 8, 2007. Accepted August 21, 2008

\section{Introduction}

Heart failure (HF) is a common endpoint for many forms of cardiovascular disease and a significant cause of morbidity and mortality (1). The development of end-stage HF often involves an initial insult to the myocardium that reduces cardiac output and leads to a compensatory increase in sympathetic nervous system activity, which ultimately leads to cardiac dysfunction and remodeling $(2,3)$. In fact, cardiac hypertrophy in HF is associated with poor prognosis and constitutes an important independent risk factor for death (4). Thus, therapies that counteract the 
deleterious effect of sympathetic hyperactivity on cardiac function and structure in $\mathrm{HF}$ are promising for the treatment of HF.

Pharmacological therapy of HF involves the use of $\beta$ blockers that recognizably protect the myocardium from excessive adrenergic stimulation (5). In fact, we have previously observed that carvedilol treatment of HF patients decreases sympathetic activity measured directly by microneurography performed on the peroneal nerve (6). Although $\beta$-blockers are known for their positive impact on cardiac function and their ability to reverse remodeling (7), they fail to improve forearm blood flow and overall functional capacity of HF patients (6). However, when $\beta$ blocker therapy is associated with exercise training there is an increase in forearm blood flow and exercise capacity (8).

Exercise training is considered to be an adjunctive therapeutic strategy for HF (9), since it improves exercise tolerance, endothelial function, and biochemical and structural parameters of skeletal muscles (10-12). Indeed, exercise training is effective in reducing a number of cardiovascular risk factors $(13,14)$. However, the effects of exercise training on left ventricular structure and function are not completely understood. In fact, several studies in humans and animals have reported contradictory effects (15-19).

In the present study, we used a genetic model of sympathetic hyperactivity-induced HF in mice to determine the isolated effect of $\beta$-blockers and exercise training on cardiac structure and function, and overall functional capacity. It is important to point out that exercise training and $\beta$-blocker treatment were started before mice presented signs of HF in order to observe the potential preventive role of both therapies. We hypothesized that both exercise training and $\beta$-blocker treatment would improve cardiac function in HF mice. However, exercise training benefits would be related to an improved exercise tolerance and skeletal muscle capillarity density while $\beta$-blocker therapy benefits would be associated with reduced ventricular fibrosis and hypertrophy.

\section{Material and Methods}

\section{Animal care}

Male wild-type (WT) and congenic $\alpha_{2 A} / \alpha_{2 C}$-adrenoceptor knockout $\left(\alpha_{2 \mathrm{~A}} / \alpha_{2 \mathrm{C}} \mathrm{ArKO}\right)$ mice with $\mathrm{C} 57 \mathrm{BI} / \mathrm{J}$ genetic background, 3 to 5 months of age, were studied. At 3 months of age, $\alpha_{2 A} / \alpha_{2 C}$ ArKO mice display preserved cardiac function with no signs of lung water retention. In contrast, at 5 months of age, cardiomyopathy is associated with a modest but significantly increased water retention in lungs (20), consistent with pulmonary edema. Genotypes were determined by polymerase chain reaction on genom- ic DNA obtained from tail biopsies using primers to detect the intact and disrupted genes.

Mice were maintained in a light- (12-h light cycle) and temperature- $\left(22^{\circ} \mathrm{C}\right)$ controlled environment and were fed a pellet rodent diet (Nuvital Nutrientes S/A, Brazil) ad libitum and had free access to water. WT and $\alpha_{2 \mathrm{~A}} / \alpha_{2 \mathrm{C}} \mathrm{ArKO}$ mice were randomly assigned to control $(\mathrm{N}=45)$, exercisetrained $(\mathrm{N}=33)$ and carvedilol-treated $(\mathrm{N}=29)$ groups. This study was carried out in accordance with Ethical Principles of animal research adopted by the Brazilian College of Animal Experimentation (www.cobea.org.br). In addition, this study was approved by the University of São Paulo Ethics Committee (CEP \#004).

\section{Procedures and measurements}

Drug treatment and exercise training protocol. Drug treatment consisted of 8 weeks of carvedilol by gavage (38 $\mathrm{mg} / \mathrm{kg}$ per day; Baldacci S.A., Brazil). The dose of carvedilol was optimized to achieve comparable heart rate (HR) levels as observed in the WT control group.

Exercise training consisted of swimming sessions 5 days/week with gradually increased duration up to $60 \mathrm{~min}$, for 8 weeks in a warmed water $\left(30-32^{\circ} \mathrm{C}\right)$ swimming apparatus adapted for mice (21). The training sessions were performed during the dark part of light cycle of the mice. This swimming protocol has been characterized as low to moderate intensity and associated with improved muscle oxidative capacity and resting bradycardia (21).

Graded treadmill exercise test. Exercise capacity, estimated by total distance run, was evaluated using a graded treadmill exercise protocol for mice. After being adapted to treadmill exercises for 1 week (10-min exercise session), mice were placed in the exercise lane and allowed to acclimatize for at least $30 \mathrm{~min}$. Exercise began at $6 \mathrm{~m} / \mathrm{min}$ with no grade and increased by $3 \mathrm{~m} / \mathrm{min}$ every $3 \mathrm{~min}$ thereafter until exhaustion. The graded treadmill exercise test was performed with both WT and $\alpha_{2 \mathrm{~A}} / \alpha_{2 \mathrm{C}} \mathrm{ArKO}$ mice before and after the experimental period.

Cardiovascular measurements. HR was determined non-invasively using a computerized tail-cuff system (BP 2000 Visitech Systems, USA) described elsewhere (22). Mice were acclimatized to the apparatus during daily sessions for 6 days, one week before starting the experimental period. HR measurements were obtained serially in WT and $\alpha_{2 \mathrm{~A}} / \alpha_{2 \mathrm{C}} \mathrm{ArKO}$ mice once a week throughout the 8-week experiment.

Non-invasive cardiac function was assessed by twodimensional guided M-mode echocardiography in halothane-anesthetized WT and $\alpha_{2 \mathrm{~A}} / \alpha_{2 \mathrm{C}}$ ArKO mice, before and after the experimental period. Briefly, mice were positioned in the supine position with front paws wide open, 
and an ultrasound transmission gel was applied to the precordium. Transthoracic echocardiography was performed using an Acuson Sequoia model 512 echocardiographer (Siemens, USA) equipped with a 14-MHz linear transducer. Left ventricle systolic function was estimated by fractional shortening as follows: Fractional shortening $(\%)=[($ LVEDD-LVESD $) / L V E D D] \times 100$ where, LVEDD = left ventricular end-diastolic dimension, and LVESD = left ventricular end-systolic dimension.

\section{Structural analysis}

Twenty-four hours after the last drug administration or exercise-training session, all mice were killed and their tissues harvested. The heart was stopped at diastole $(\mathrm{KCl}$, $14 \mathrm{mM}$ ) and dissected to obtain the left ventricle, which corresponds to the remaining organ upon removal of both atria and free wall of the right ventricle. For morphometric analysis, left ventricle samples obtained from the free wall, at the level of papillary muscle, were fixed in $4 \%$ buffered formalin and embedded in paraffin, cut in 4- $\mu \mathrm{m}$ sections and subsequently stained with hematoxylin and eosin. Two randomly selected sections from each animal were visualized by light microscopy using an objective with a calibrated magnification (400X). Myocytes with visible nucleus and intact cellular membranes were chosen for diameter determination. The width of an individually isolated cardiomyocyte displayed on a viewing screen was manually traced across the middle of the nuclei with a digitizing pad and determined by a computer-assisted image analysis system (Quantimet 520; Cambridge Instruments, UK). For each section, approximately 15 visual fields were analyzed.

Quantification of left ventricular fibrosis was achieved by Sirius red staining. Two randomly selected sections from each animal were visualized by light microscopy using an objective with a calibrated magnification of 200X. The interstitial collagen area was quantified by a computer-assisted image analysis system (Quantimet 520; Cambridge Instruments, USA). Approximately 5 visual fields were analyzed for each animal.

Capillary-to-fiber ratio was quantified in gastrocnemius muscle after periodic acid-Schiff staining. Two randomly selected sections from each animal were visualized by light microscopy using an objective with a calibrated magnification of 400X. The number of capillaries per fiber was analyzed in a computer-assisted morphometric system (Leica Quantimet 520, Cambridge Instruments). Approximately 15 visual fields were analyzed for each animal.

\section{Statistical analysis}

Data are reported as means \pm SEM. Two-way ANOVA for repeated measurements with post hoc testing by Tukey (Statistica software, StatSoft, Inc., USA) was used to compare the effect of treatment (control, exercise-trained and carvedilol-treated) and genotype (WT and $\alpha_{2 \mathrm{~A}} / \alpha_{2 \mathrm{C}}$ ArKO) on HR measurements throughout the experimental period. Two-way ANOVA with post hoc testing by Tukey was used to compare the effect of treatment (control, exercise-trained and carvedilol-treated) and genotype (WT and $\alpha_{2 \mathrm{~A}} / \alpha_{2 \mathrm{C}}$ ArKO) on distance run, capillary density, fractional shortening and cardiac structure analysis. Statistical significance was set at $P<0.05$.

\section{Results}

Effect of exercise training and carvedilol treatment on exercise tolerance, heart rate and capillary density

Before starting the experiment (at 3 months of age), $\alpha_{2 \mathrm{~A}} / \alpha_{2 \mathrm{C}} \mathrm{ArKO}$ mice presented exercise capacity similar to WT mice (428 \pm 99 vs $425 \pm 107 \mathrm{~m}$, respectively). However, after 8 weeks of experiment (at 5 months of age), $\alpha_{2 \mathrm{~A}}$ l $\alpha_{2 \mathrm{C}}$ ArKO mice displayed exercise intolerance compared with age-matched WT mice (Figure 1A). Exercise training prevented exercise intolerance in $\alpha_{2 \mathrm{~A}} / \alpha_{2 \mathrm{C}} \mathrm{ArKO}$ mice, and increased exercise tolerance in WT mice (Figure 1A). In contrast, carvedilol treatment had no impact on exercise tolerance in either WT or $\alpha_{2 \mathrm{~A}} / \alpha_{2 \mathrm{C}} \mathrm{ArKO}$ mice. $\alpha_{2 \mathrm{~A}} / \alpha_{2 \mathrm{C}} \mathrm{ArKO}$ mice displayed baseline tachycardia when compared with age-matched WT mice (Figure 1B). Both exercise training and carvedilol treatment significantly decreased baseline $\mathrm{HR}$ in $\alpha_{2 \mathrm{~A}} / \alpha_{2 \mathrm{C}}$ ArKO mice reaching WT control levels from the 5 th week of experiment onward (Figure 1B). The magnitude of $H R$ reduction was similar in both trained and carvedilol-treated $\alpha_{2 A} / \alpha_{2 C}$ ArKO groups. As expected, exercise training and carvedilol treatment also reduced baseline HR in WT mice.

$\alpha_{2 \mathrm{~A}} / \alpha_{2 \mathrm{C}} \mathrm{ArKO}$ mice displayed capillary rarefaction when compared with age-matched WT mice (Figure 1C). Exercise training significantly prevented capillary rarefaction in gastrocnemius muscle of $\alpha_{2 \mathrm{~A}} / \alpha_{2 \mathrm{C}} \mathrm{ArKO}$ mice, and significantly increased the capillary-to-fiber ratio in WT mice. Carvedilol treatment had no effect on capillary density in either WT or $\alpha_{2 \mathrm{~A}} / \alpha_{2 \mathrm{C}}$ ArKO mice (Figure 1C).

\section{Effect of exercise training and carvedilol treatment on} fractional shortening and cardiac structure

At the beginning of the experiment (at 3 months of age), there was no difference between WT and $\alpha_{2 \mathrm{~A}} / \alpha_{2 \mathrm{C}} \mathrm{ArKO}$ mice in fractional shortening $(20.9 \pm 1.4$ vs $18.0 \pm 0.5 \%)$, left ventricle diastolic diameter $(3.72 \pm 0.1$ vs $3.60 \pm 0.1$ $\mathrm{mm})$, and left ventricle posterior wall thickness $(0.60 \pm$ 0.001 vs $0.60 \pm 0.001 \mathrm{~mm}$ ). However, after 8 weeks (at 5 
months of age), $\alpha_{2 \mathrm{~A}} / \alpha_{2 \mathrm{C}}$ ArKO mice displayed systolic dysfunction when compared with age-matched WT mice (Figure $2 \mathrm{~A}$ ) and a trend toward increased left ventricle diastolic diameter $(3.80 \pm 0.07$ vs $3.92 \pm 0.03 \mathrm{~mm}, \mathrm{P}=0.08)$. Both exercise training and carvedilol treatment prevented the systolic dysfunction in $\alpha_{2 \mathrm{~A}} / \alpha_{2 \mathrm{C}} \mathrm{ArKO}$ mice by increasing fractional shortening towards WT mice values (Figure 2A). In addition, while exercise training significantly increased fractional shortening in WT mice, carvedilol treatment had no impact on cardiac contractility (Figure $2 \mathrm{~A}$ ).

Quantitative morphometric analysis showed that cardiac myocyte cross-sectional diameter was significantly
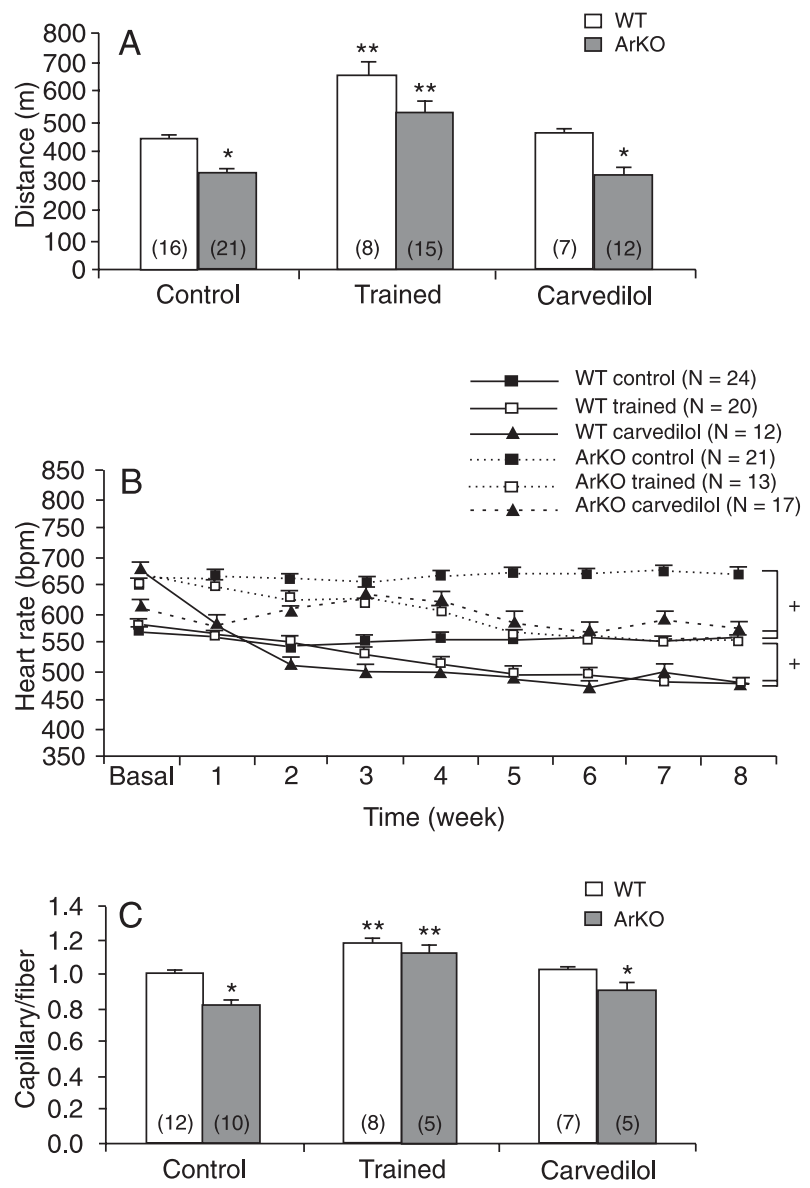

Figure 1. Effect of exercise training and carvedilol administration on exercise capacity measured as maximal distance run $(A)$, heart rate $(B)$ and capillary-to-fiber ratio in gastrocnemius muscle (C) evaluated in wild-type (open columns, WT) and $\alpha_{2 \mathrm{~A}} / \alpha_{2} \mathrm{~A}$ ArKO (filled columns, ArKO) mice. The exercise training significantly improved distance run and capillary-to-fiber ratio while it decreased heart rate in $\alpha_{2 \mathrm{~A}} / \alpha_{2} \mathrm{CArKO}$ mice. In contrast, carvedilol treatment had no effect on exercise capacity and capillary density, but reduced baseline heart rate in $\alpha_{2 \mathrm{~A}} / \alpha_{2 \mathrm{C}} \mathrm{ArKO}$ mice. As expected, trained WT mice displayed resting bradycardia and increased exercise capacity and capillary density. The number in parentheses indicate the number of mice in each group. Data are reported as means \pm SEM. ${ }^{*} \mathrm{P}<0.05$ compared to WT control group; ${ }^{* *} \mathrm{P}<0.05$ compared to respective control group; ${ }^{+} P<0.05$ compared to respective control group from the 4th week to the end of experimental period.
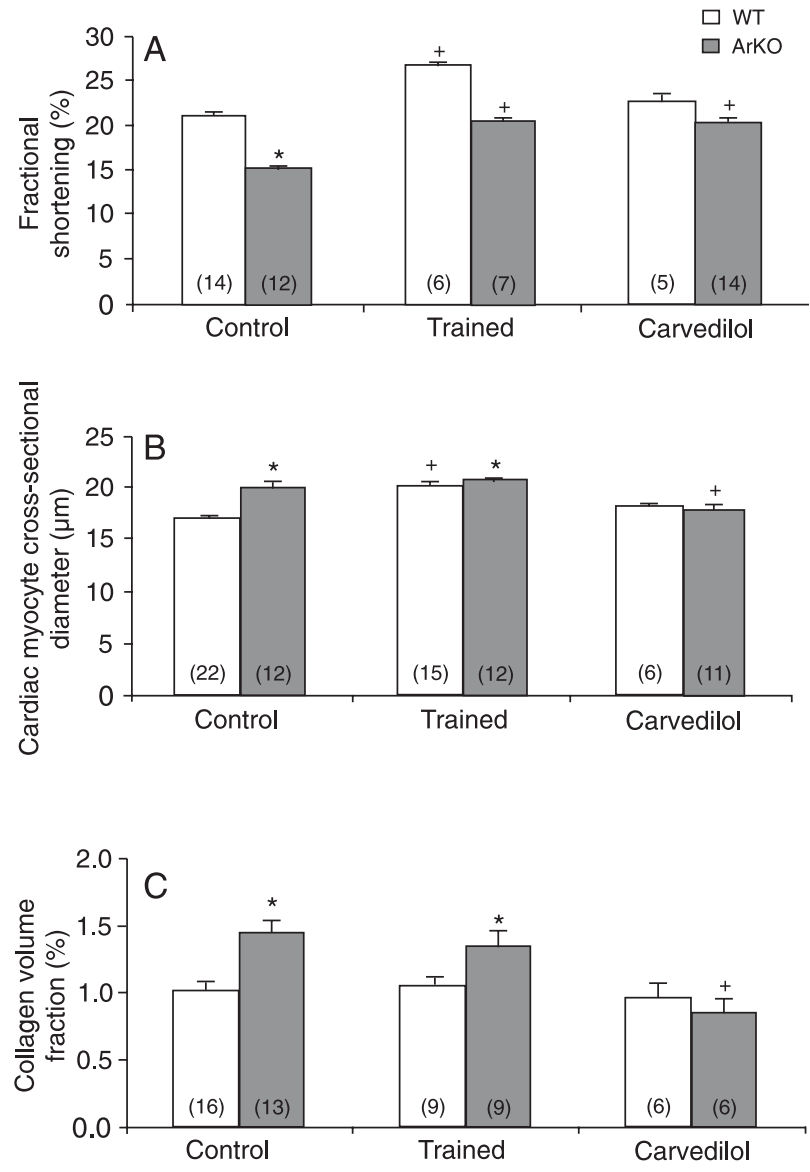

Figure 2. Effect of exercise training and carvedilol administration on fractional shortening used as an index of systolic function (A), cardiac myocyte cross-sectional diameter (B) and cardiac collagen volume fraction $(\mathrm{C})$ evaluated in wild-type (open columns, WT) and $\alpha_{2 A} / \alpha_{2}$ ArKO (filled columns, ArKO) mice. The exercise training and carvedilol treatment significantly increased fractional shortening in $\alpha_{2 \mathrm{~A}} / \alpha_{2} \mathrm{~A}$ ArKO mice. However, only carvedilol treatment prevented cardiac remodeling by reducing cardiac myocyte cross-sectional diameter and collagen volume fraction in $\alpha_{2 \mathrm{~A}} / \alpha_{2 \mathrm{C}} \mathrm{ArKO}$ mice. The number in parentheses indicate the number of mice in each group. Data are reported as means \pm SEM. ${ }^{*} \mathrm{P}<0.05$ compared to WT control group; ${ }^{+} \mathrm{P}<0.05$ compared to respective control group. 
greater in $\alpha_{2 \mathrm{~A}} / \alpha_{2 \mathrm{C}} \mathrm{ArKO}$ mice compared with WT control mice (Figure 2B). Exercise training had no effect on cardiac myocyte cross-sectional diameter in $\alpha_{2 A} / \alpha_{2 C}$ ArKO mice but increased it in WT mice. Carvedilol significantly reduced cardiac myocyte width in $\alpha_{2 A} / \alpha_{2 C} A r K O$ mice. The reduction of cardiac myocyte cross-sectional diameter in carvedilol-treated $\alpha_{2 \mathrm{~A}} / \alpha_{2 \mathrm{C}} \mathrm{ArKO}$ mice was so remarkable that it reached WT control mice levels (Figure 2B).

Increased cardiac myocyte cross-sectional diameter in $\alpha_{2 \mathrm{~A}} / \alpha_{2 \mathrm{C}} \mathrm{ArKO}$ mice was paralleled by ventricular fibrosis, represented by an increase of $45 \%$ in cardiac collagen volume fraction of $\alpha_{2 \mathrm{~A}} / \alpha_{2 \mathrm{C}}$ ArKO mice compared with WT control mice (Figure 2C). Exercise training did not change cardiac collagen volume fraction in either WT or $\alpha_{2 \mathrm{~A}}$ l $\alpha_{2 \mathrm{C}}$ ArKO mice. In contrast, carvedilol treatment significantly reduced ventricular fibrosis in $\alpha_{2 \mathrm{~A}} / \alpha_{2 \mathrm{C}} \mathrm{ArKO}$ mice, but cardiac collagen volume fraction of carvedilol-treated WT mice remained unchanged (Figure $2 \mathrm{C}$ ).

\section{Discussion}

The main findings of the present study were that exercise training and $\beta$-blocker therapy improved ventricular function of HF mice similarly. However, the effect of exercise training was mainly associated with improved exercise tolerance and skeletal muscle capillary density while $\beta$-blocker therapy had a greater impact on cardiac structure.

Exercise training is emerging as an adjuvant nonpharmacological therapy for HF (9) and the benefits of exercise training on HF are related to improved function of both cardiac and skeletal muscles (10-12,23). In the present investigation, we observed improved fractional shortening paralleled by an increased gastrocnemius capillary density in exercise-trained $\alpha_{2 A} / \alpha_{2 C}$ ArKO mice, which ultimately led to improved exercise tolerance. The importance of these results is striking because exercise intolerance is associated with a poor prognosis and reduced quality of life in HF patients (24). Indeed, an unacceptable burden of disability and unrelieved symptoms still remain even in optimally medically treated HF patients $(25,26)$.

The mechanisms underlying the improved cardiac function and gastrocnemius capillary density in exercise-trained $\alpha_{2 \mathrm{~A}} / \alpha_{2 \mathrm{C}} \mathrm{ArKO}$ mice were not investigated in this study, but we have previously demonstrated an improved cardiac myocyte $\mathrm{Ca}^{2+}$ handling after exercise training in $\alpha_{2 \mathrm{~A}}$ l $\alpha_{2 \mathrm{C}}$ ArKO mice $(27,28)$. Indeed, increased expression of vascular growth factors seems to be involved in angiogenesis induced by exercise training (29).

The effects of exercise training on left ventricular remodeling in HF are under investigation (15). Exercise training had no detectable impact on left ventricular fibrosis of $\alpha_{2 A} / \alpha_{2 C}$ ArKO mice. In fact, previous studies demonstrated little effect of exercise training in total cardiac collagen fraction (30). Likewise, exercise training did not change the increased cardiac myocyte width of $\alpha_{2 A} / \alpha_{2 C} A r K O$ mice. However, we cannot exclude the possibility that swimming training might preclude pathological hypertrophy, since it induces physiological remodeling that is associated with improved cardiac function. In fact, as we observed in WT mice, swimming exercise training led to a physiological hypertrophy, which we showed previously to be associated with beneficial cardiac effects (21).

In contrast to exercise training, carvedilol treatment had no effect on gastrocnemius capillary density and exercise tolerance in $\alpha_{2 A} / \alpha_{2 C} A r K O$ mice. This response was somehow expected, since carvedilol is a non-selective $\beta$ blocker with transient $\alpha_{1}$-adrenoceptor blockade, which could reduce skeletal muscle vasodilatation and preclude improvement in exercise tolerance.

The benefits of carvedilol were mainly due to its effect on cardiac function and structure. In fact $\beta$-blockers yield impressive benefits on cardiac contractility of patients with chronic heart failure (17). In the present study, we observed an increased fractional shortening in carvediloltreated $\alpha_{2 A} / \alpha_{2 C}$ ArKO mice associated with reduced left ventricular fibrosis and cardiac myocyte hypertrophy. These observations suggest that carvedilol prevents cardiac remodeling in $\alpha_{2 \mathrm{~A}} / \alpha_{2 \mathrm{C}}$ ArKO mice. The mechanisms involved in reduced cardiac fibrosis and hypertrophy of carvediloltreated $\alpha_{2 \mathrm{~A}} / \alpha_{2 \mathrm{C}}$ ArKO mice were not examined in the present investigation; however, reduced cardiac angiotensin II levels might be involved. In fact, we have recently demonstrated that $\alpha_{2 A} / \alpha_{2 C}$ ArKO mice display increased cardiac angiotensin II levels associated with ventricular fibrosis reversed partially by losartan treatment (20).

The present results demonstrate that both exercise training and carvedilol therapy improve, to the same extent, the ventricular function in a genetic model of sympathetic hyperactivity-induced heart failure in mice. However, while the benefits of exercise training are mainly associated with increased aerobic capacity and capillary density of skeletal muscle, carvedilol benefits were mainly due to its effect on cardiac structure. These results indicate that studies are needed to determine the efficacy of exercise training associated with $\beta$-blockers for treatment of heart failure therapy.

\section{Acknowledgments}

The authors acknowledge Baldacci S.A. for donating carvedilol for this study. 


\section{References}

1. Thom T, Haase N, Rosamond W, Howard VJ, Rumsfeld J, Manolio T, et al. Heart disease and stroke statistics - 2006 update: a report from the American Heart Association Statistics Committee and Stroke Statistics Subcommittee. Circulation 2006; 113: e85-e151.

2. Packer M. The neurohormonal hypothesis: a theory to explain the mechanism of disease progression in heart failure. J Am Coll Cardiol 1992; 20: 248-254.

3. Bristow MR. The adrenergic nervous system in heart failure. N Engl J Med 1984; 311: 850-851.

4. Benjamin EJ, Levy D. Why is left ventricular hypertrophy so predictive of morbidity and mortality? Am J Med Sci 1999; 317: 168-175.

5. Mann DL. Basic mechanisms of disease progression in the failing heart: the role of excessive adrenergic drive. Prog Cardiovasc Dis 1998; 41: 1-8.

6. De Matos LD, Gardenghi G, Rondon MU, Soufen HN, Tirone AP, Barretto AC, et al. Impact of 6 months of therapy with carvedilol on muscle sympathetic nerve activity in heart failure patients. J Card Fail 2004; 10: 496-502.

7. Bristow MR, Roden RL, Lowes BD, Gilbert EM, Eichhorn EJ. The role of third-generation beta-blocking agents in chronic heart failure. Clin Cardiol 1998; 21: I-3-I-13.

8. Fraga R, Franco FG, Roveda F, de Matos LN, Braga AM, Rondon $\mathrm{MU}$, et al. Exercise training reduces sympathetic nerve activity in heart failure patients treated with carvedilol. Eur J Heart Fail 2007; 9: 630-636.

9. Klecha A, Kawecka-Jaszcz K, Bacior B, Kubinyi A, Pasowicz M, Klimeczek $P$, et al. Physical training in patients with chronic heart failure of ischemic origin: effect on exercise capacity and left ventricular remodeling. Eur J Cardiovasc Prev Rehabil 2007; 14: 85-91.

10. You Fang Z, Marwick TH. Mechanisms of exercise training in patients with heart failure. Am Heart $J$ 2003; 145: 904911.

11. McKelvie RS, Teo KK, Roberts R, McCartney N, Humen D, Montague $\mathrm{T}$, et al. Effects of exercise training in patients with heart failure: the Exercise Rehabilitation Trial (EXERT). Am Heart J 2002; 144: 23-30.

12. Corra U, Mezzani A, Giannuzzi P, Tavazzi L. Chronic heart failure-related myopathy and exercise training: a developing therapy for heart failure symptoms. Curr Probl Cardiol 2003; 28: 521-547.

13. Emter CA, McCune SA, Sparagna GC, Radin MJ, Moore $\mathrm{RL}$. Low-intensity exercise training delays onset of decompensated heart failure in spontaneously hypertensive heart failure rats. Am J Physiol Heart Circ Physiol 2005; 289: $\mathrm{H} 2030-\mathrm{H} 2038$.

14. Powers SK, Lennon SL, Quindry J, Mehta JL. Exercise and cardioprotection. Curr Opin Cardiol 2002; 17: 495-502.

15. de Waard MC, van der Velden J, Bito V, Ozdemir S, Biesmans L, Boontje NM, et al. Early exercise training normalizes myofilament function and attenuates left ventricular pump dysfunction in mice with a large myocardial infarction. Circ Res 2007; 100: 1079-1088.

16. Giannuzzi P, Tavazzi L, Temporelli PL, Corra U, Imparato A, Gattone M, et al. Long-term physical training and left ventricular remodeling after anterior myocardial infarction: results of the Exercise in Anterior Myocardial Infarction (EAMI) trial. EAMI Study Group. J Am Coll Cardiol 1993; 22: 1821-1829.

17. Giannuzzi P, Temporelli PL, Corra U, Tavazzi L. Antiremodeling effect of long-term exercise training in patients with stable chronic heart failure: results of the Exercise in Left Ventricular Dysfunction and Chronic Heart Failure (ELVDCHF) Trial. Circulation 2003; 108: 554-559.

18. Kubo N, Ohmura N, Nakada I, Yasu T, Katsuki T, Fujii M, et al. Exercise at ventilatory threshold aggravates left ventricular remodeling in patients with extensive anterior acute myocardial infarction. Am Heart J 2004; 147: 113-120.

19. Sullivan MJ, Higginbotham MB, Cobb FR. Exercise training in patients with severe left ventricular dysfunction. Hemodynamic and metabolic effects. Circulation 1988; 78: 506-515.

20. Ferreira JC, Bacurau AV, Evangelista FS, Coelho MA, Oliveira EM, Casarini DE, et al. The role of local and systemic renin angiotensin system activation in a genetic model of sympathetic hyperactivity-induced heart failure in mice. Am J Physiol Regul Integr Comp Physiol 2008; 294: R26-R32.

21. Evangelista FS, Brum PC, Krieger JE. Duration-controlled swimming exercise training induces cardiac hypertrophy in mice. Braz J Med Biol Res 2003; 36: 1751-1759.

22. Johns C, Gavras I, Handy DE, Salomao A, Gavras H. Models of experimental hypertension in mice. Hypertension 1996; 28: 1064-1069.

23. Belardinelli R, Georgiou D, Cianci G, Purcaro A. Randomized, controlled trial of long-term moderate exercise training in chronic heart failure: effects on functional capacity, quality of life, and clinical outcome. Circulation 1999; 99: 11731182.

24. Pozehl B, Duncan K, Hertzog M. The effects of exercise training on fatigue and dyspnea in heart failure. Eur J Cardiovasc Nurs 2008; 7: 127-132.

25. McAlister FA, Lawson FM, Teo KK, Armstrong PW. A systematic review of randomized trials of disease management programs in heart failure. Am J Med 2001; 110: 378-384.

26. Anonymous. Recommendations for exercise training in chronic heart failure patients. Eur Heart $J$ 2001; 22: 125135.

27. Rolim NP, Medeiros A, Rosa KT, Mattos KC, Irigoyen MC, Krieger EM, et al. Exercise training improves the net balance of cardiac $\mathrm{Ca}^{2+}$ handling protein expression in heart failure. Physiol Genomics 2007; 29: 246-252.

28. Medeiros A, Rolim NP, Oliveira RS, Rosa KT, Mattos KC, Casarini DE, et al. Exercise training delays cardiac dysfunction and prevents calcium handling abnormalities in sympathetic hyperactivity-induced heart failure mice. J Appl Physiol 2008; 104: 103-109.

29. Amaral SL, Papanek PE, Greene AS. Angiotensin II and VEGF are involved in angiogenesis induced by short-term exercise training. Am J Physiol Heart Circ Physiol 2001; 281: H1163-H1169.

30. Thomas DP, Zimmerman SD, Hansen TR, Martin DT, McCormick RJ. Collagen gene expression in rat left ventricle: interactive effect of age and exercise training. $J$ Appl Physiol 2000; 89: 1462-1468. 